\title{
The constructal-law physics of why swimmers must spread their fingers and toes
}

\author{
S. Lorente ${ }^{1}$, E. Cetkin ${ }^{2}$, T. Bello-Ochende ${ }^{3}$, J. P. Meyer ${ }^{3}$ and A. Bejan ${ }^{2}$ \\ ${ }^{1}$ Université de Toulouse, INSA, Laboratoire Matériaux et Durabilité des Constructions, 135, Avenue de Rangueil, \\ F-31077 Toulouse Cedex 04, France \\ ${ }^{2}$ Duke University, Department of Mechanical Engineering and Materials Science, Box 90300, Durham, NC 27708- \\ 0300, USA \\ ${ }^{3}$ University of Pretoria, Department of Mechanical and Aeronautical Engineering, Pretoria 0002, South Africa
}

\section{Highlights}

- The total force is $53 \%$ greater when the fingers are spaced optimally.

- The optimal spacing is twice the boundary layer thickness of one finger.

- The speed advantage comes from the greater force, which lifts more mass above water.

- The theoretical predictions are confirmed by computational fluid dynamics simulations.

\begin{abstract}
Here we show theoretically that swimming animals and athletes gain an advantage in force and speed by spreading their fingers and toes optimally. The spacing between fingers must betwice the thickness of the boundary layer around one finger. This theoretical prediction is confirmed by computational fluid dynamics simulations of flow across two and four cylinders of diameter D. The optimal spacing is in the range $0.2 \mathrm{D}-0.4 \mathrm{D}$, and decreases slightly as the Reynolds number (Re) increases from 20 to 100 . The total force exerted by optimally spacing two cylinders exceeds by 53 percent the total force of two cylinders with no spacing when $\operatorname{Re}=20$. These design features hold for both time-dependent and steady-state flows.
\end{abstract}


Keywords: Swimming, Animal locomotion, Constructal law, Webbed feet, Sports evolution.

\section{Introduction}

The sport of competitive swimming has evolved measurably during its 100 years of modern history. Record speeds have increased steadily, and so have the principal body measurements of the record breakers: the mass, the height and the slenderness of the body shape (Charles and Bejan, 2009). In brief, bigger and taller means faster, and this trend coincides not only with the mass-speed scaling of all animals with locomotion (swimmers, runner and flies; cf. Bejan and Marden, 2006) but also with the measurable evolution of the sport of speed running. The scientific contribution that sport evolution makes is that it provides a laboratory in which we can observe the phenomenon of evolution in our life time.

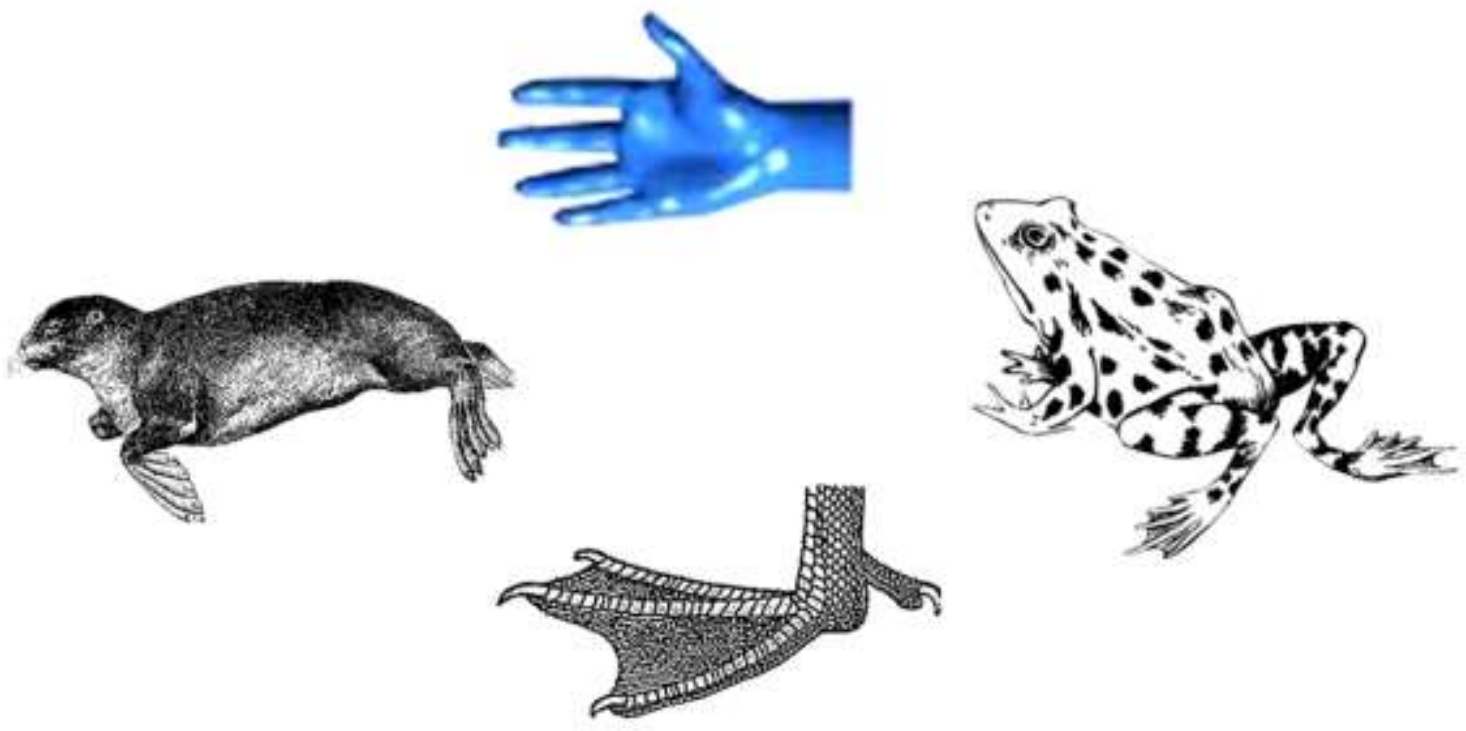

Fig. 1. Examples of palms and feet of aquatic animals and swimming humans.

In this paper we identify the physics origin of the emergence of paddle-shaped feet and palms 
in animals that swim (Fig. 1). This feature of animal design is considered understood, based on the argument that pushing the water with a larger paddle makes swimming more efficient. Upon closer inspection, however, this explanation is questionable, because a larger paddle means a larger force exerted on the surrounding water body, not a higher efficiency. This body of work is just one application of the constructal law to the evolution of design in nature (e.g., Miguel, 2006; Reis, 2006a; Reis et al., 2004), which was reviewed in Hoppeler and Weibel (2005), Reis (2006b) and Bejan and Lorente (2010).

The fundamental question for theoretical biology is why a paddling body should be advantaged by a paddle that exerts a greater force. We propose to answer this question by using the sport of competitive swimming. We focus on the shape of the human hand during speed swimming. Athletes today are being trained to swim with their fingers spread slightly. All competitive swimmers swim this way because this configuration generates greater speed (note: speed, not force, because the direction of this evolutionary design is toward speed). Further evidence, also empirical, is offered by computational fluid dynamics simulations showing that a hand with fingers spread slightly exerts a greater force, roughly 5-10 percent greater than when the fingers are held tight, and greater than when the fingers are spread far apart (Minetti et al., 2009; Marinho et al., 2010).

Recent theoretical advances (Charles and Bejan, 2009; Bejan et al., 2010) showed that better performances in speed swimming are predictable, because:

(1) Swimming is the motion of surfing on the water wave generated by the swimmer,

(2) Bigger waves travel horizontally faster, and

(3) Bigger (i. e. longer) swimmers can raise their torsos higher above the water line, in order to generate bigger waves and greater speeds.

The new connection that follows from (1) - (3) is that in order to raise the body higher above the 
water line (i.e., in order to lift a larger weight), the swimmer must be able to push the water downward with a greater force. Speed in sports comes from this principle (Charles and Bejan, 2009), and this holds for all swimming animals as well (Bejan and Marden, 2006). Lifting a larger weight requires a larger downward force, and this is why larger paddles (spread fingers and toes, with web or without) is a common design in evolutionary biology.

The theoretical analysis presented in this paper shows that an optimal (and slight) finger spacing exists, such that the total force is maximal. This prediction is validated based on computational simulation of fluid flow around and through parallel cylinders in cross flow. Although the more recent computational fluid dynamic simulation of swimming are based on realistic facsimiles of the human hand, arm and body (e. g., Bixler and Riewald, 2002; Rouboa et al., 2006, Kudo et. al., 2008), in this study we use a simple model consisting of parallel cylinders in order to identify the correct scales and scaling rules of the spread-fingers configuration. To establish this understanding theoretically (i.e. as a prediction) is important because the "optimal" finger spacing was mentioned in the literature ( e. g., Minetti et al., 2009) but it was neither optimized nor predicted.

\section{The existence of the optimal spacing}

The fact that an optimal spacing between fingers should exist can be anticipated based on constructal theory (Bejan and Lorente, 2008, ch. 3). We model each finger as a cylinder placed in cross flow. The drag force exerted by a uniform flow $\left(\mathrm{V}_{\infty}\right)$ on a perpendicular cylinder of diameter $\mathrm{Y}$ is

$$
\mathrm{F}=\mathrm{CYL} \frac{1}{2} \rho \mathrm{V}_{\infty}^{2}
$$

Note the frontal area YL seen by the approaching stream, the cylinder length L, and the fluid 
density $\rho$. The coefficient $C$ decreases with the Reynolds number $\operatorname{Re}_{\mathrm{Y}}=\mathrm{V}_{\infty} \mathrm{Y} / \nu$ (with $v$ the kinematic viscosity), however, it is essentially constant when $\operatorname{Re}_{\mathrm{Y}}$ is of order of $10^{2}$ or greater.

Important is the effect of the shape of the cylinder cross-section, which is shown in Fig. 2. Among the three shapes that have the same frontal area YL, the single cylinder with diameter Y has the smallest drag coefficient. This is the most "hydrodynamic" shape from among the configurations $\mathrm{C}_{1}, \mathrm{C}_{2}$ and $\mathrm{C}_{3}$. On the other hand, the least hydrodynamic is the slab of width $\mathrm{Y}$. In summary, the three shapes rank themselves in this order

$$
\mathrm{C}_{1}<\mathrm{C}_{2}<\mathrm{C}_{3}
$$

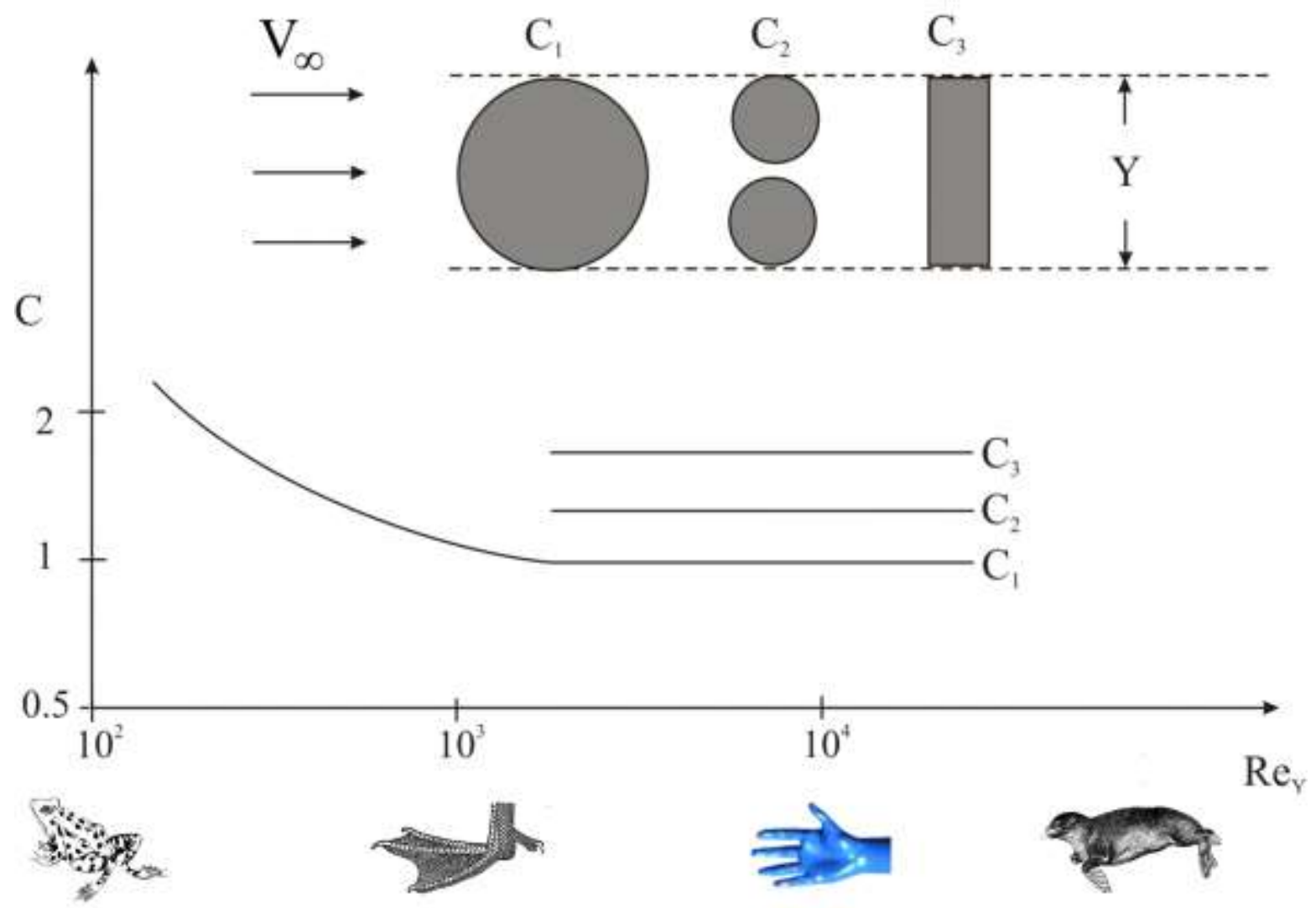

Fig. 2. The effect of cross-sectional shape on the drag coefficient for three long objects placed in cross flow.

Next, consider $n$ parallel cylinders in cross flow, as a model of paddling the water with $n$ fingers 
of diameter D. The case $n=4$ is shown in Fig. 3, which shows three configurations, in order of increasing frontal area:

(A) Cylinders stuck together, $\mathrm{Y}_{\mathrm{A}}=\mathrm{nD}$, with the total drag force

$$
\mathrm{F}_{\mathrm{A}}=\mathrm{C}_{\mathrm{A}} \mathrm{nDL} \frac{1}{2} \rho \mathrm{V}_{\infty}^{2}
$$

(B) Cylinders separated by a sufficiently small spacing (S), so that $Y_{B}=n D+(n-1) S$, and

$$
\mathrm{F}_{\mathrm{B}}=\mathrm{C}_{\mathrm{B}}[\mathrm{nD}+(\mathrm{n}-1) \mathrm{S}] \mathrm{L} \frac{1}{2} \rho \mathrm{V}_{\infty}^{2}
$$

How small a spacing $\mathrm{S}$ is "sufficiently small" will be determined later in this section.

(C) Cylinders spread far apart, each with $\mathrm{Y}=\mathrm{D}$ and the drag force $\mathrm{C}_{\mathrm{C}} \mathrm{DL} \frac{1}{2} \rho \mathrm{V}_{\infty}^{2}$,

$$
\mathrm{F}_{\mathrm{C}}=\mathrm{nC}_{\mathrm{C}} \mathrm{DL} \frac{1}{2} \rho \mathrm{V}_{\infty}^{2}
$$

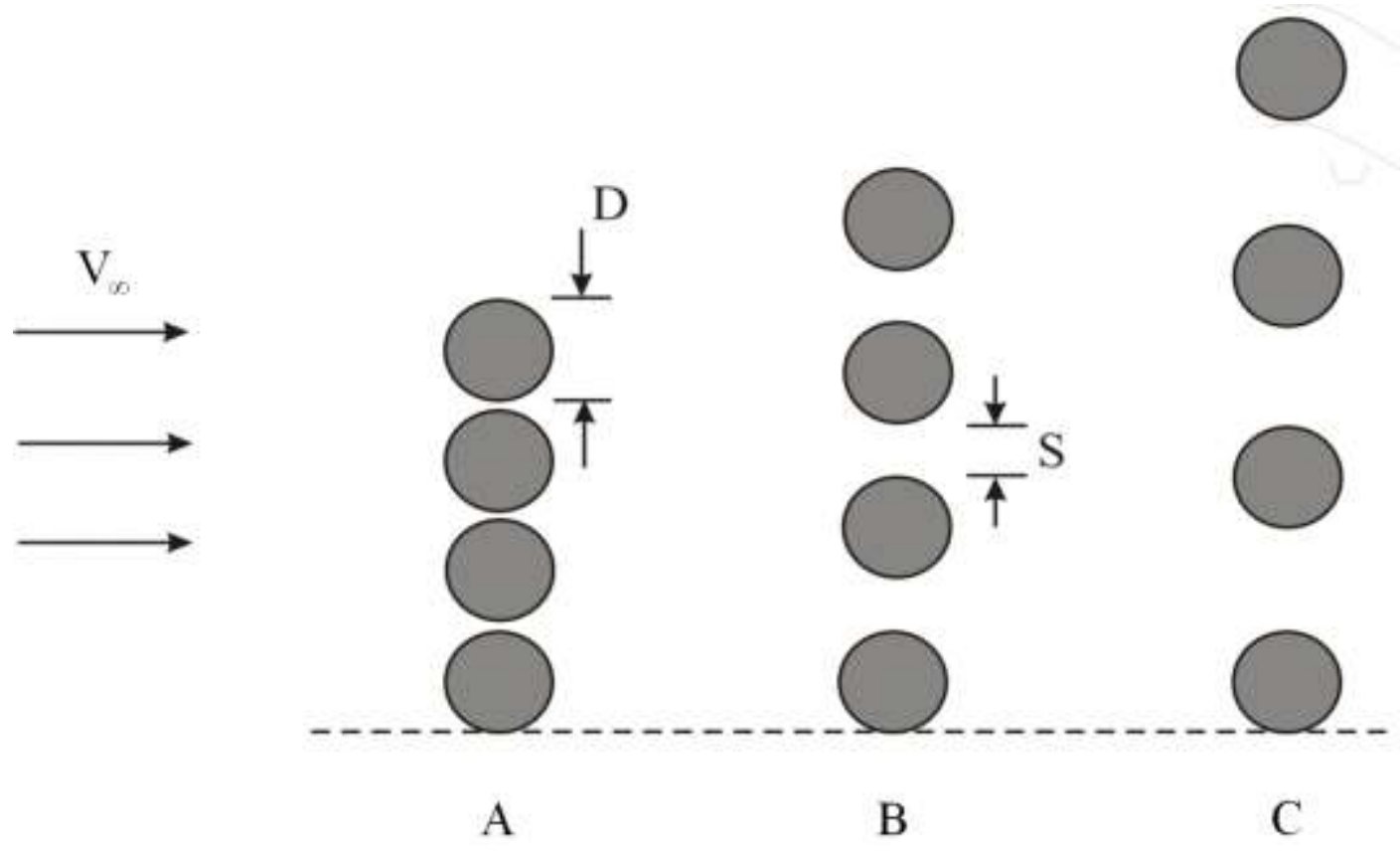

Fig. 3. Three configurations of four cylinders in cross flow: A=no spacings; $B=$ sufficiently small spacings; $C=$ large spacings. 
Finally, we make two comparisons. Dividing Eqs. (3) and (5) we obtain

$$
\frac{\mathrm{F}_{\mathrm{A}}}{\mathrm{F}_{\mathrm{C}}}=\frac{\mathrm{C}_{\mathrm{A}}}{\mathrm{C}_{\mathrm{C}}}>1
$$

The unequal sign is due to the effect shown in Fig. 2, where case A is represented by the shape $\mathrm{C}_{2}$, case $\mathrm{C}$ is represented by the shape $\mathrm{C}_{1}$, and $\mathrm{C}_{2} / \mathrm{C}_{1}>1$.

Next, we find that by dividing Eqs. (3) and (4) we obtain

$$
\frac{\mathrm{F}_{\mathrm{B}}}{\mathrm{F}_{\mathrm{A}}}=\frac{\mathrm{C}_{\mathrm{B}}}{\mathrm{C}_{\mathrm{A}}} \frac{\mathrm{nD}+(\mathrm{n}-1) \mathrm{S}}{\mathrm{nD}}>1
$$

Here the inequality is due to the fact that both factors, $\left(\mathrm{C}_{\mathrm{B}} / \mathrm{C}_{\mathrm{A}}\right.$ and the second fraction $)$ are greater than 1. Note that whereas configuration $A$ is like shape $C_{2}$ in Fig. 2, configuration $B$ is between shape $C_{2}$ and shape $C_{3}$, in other words $C_{B} / C_{A} \geq 1$.

The inequalities (6) and (7) show that the largest force corresponds to configuration B,

$$
\mathrm{F}_{\mathrm{B}}>\mathrm{F}_{\mathrm{A}}>\mathrm{F}_{\mathrm{C}}
$$

This conclusion holds for the entire Re range. Configuration B exists provided that $\mathrm{S}$ is small enough such that the stagnation pressure $\rho V_{\infty}^{2} / 2$ is maintained upstream of every $S$-wide gap. This happens when $\mathrm{S}$ does not exceed the thickness of the laminar boundary layer that surrounds each cylinder (Bejan and Lorente, 2008, ch. 3)

$$
\mathrm{S} \leq \mathrm{DRe}_{\mathrm{D}}^{-1 / 2}
$$

The largest spacing that maintains the upstream stagnation pressure, and assures the largest force has the same length scale as the thickness of the laminar boundary layer that coats one cylinder. Substituted in Eq. (7), this S estimate shows that the force advantage associated with spreading the cylinders (away from configuration A) should be of order

$$
\frac{\mathrm{F}_{\mathrm{B}}}{\mathrm{F}_{\mathrm{A}}} \leq 1+\left(1-\frac{1}{\mathrm{n}}\right) \operatorname{Re}_{\mathrm{D}}^{-1 / 2}
$$


Because $\operatorname{Re}_{\mathrm{D}}$ is of order $10^{2}$ or greater (Fig. 2), the force $F_{B}$ should be greater than $F_{A}$ by roughly 10 percent. This is a significant advantage in competing for survival among swimming animals, and for speed in sports. The predicted 10-percent force increase due to spacing the cylinders agrees in an order of magnitude sense with the increase determined based on computational fluid dynamics in Fig. 3 of Marinho et al., 2010, and Fig. 2 of Minetto et al., 2009.

\section{Numerical model and method}

The preceding theory holds for the entire Re range occupied by the most common swimmers (Fig. 1). For example, a frog-size swimmer has a finger diameter of order D $1 \mathrm{~mm}$ and stroke speed $\mathrm{V}_{\infty} \sim 0.1 \mathrm{~m} / \mathrm{s}$, which in water correspond to $\mathrm{Re} \sim 100$. For the hand of a swimming athlete, the scales are $\mathrm{D} \sim 0.01 \mathrm{~m}$ and $\mathrm{V}_{\infty} \sim 1 \mathrm{~m} / \mathrm{s}$, which correspond to $\mathrm{Re} \sim 10^{4}$. We reinforced the theory with our own computational simulations of fluid flow and forces in the Re range 20 1000. We started with the simplest configuration, which consists of just two cylinders, Fig. 4. The objective of the numerical simulations is to estimate the drag forces $\left(\mathrm{F}_{1}, \mathrm{~F}_{2}\right)$ and discover how they depend on the spacing $\mathrm{S}$. 


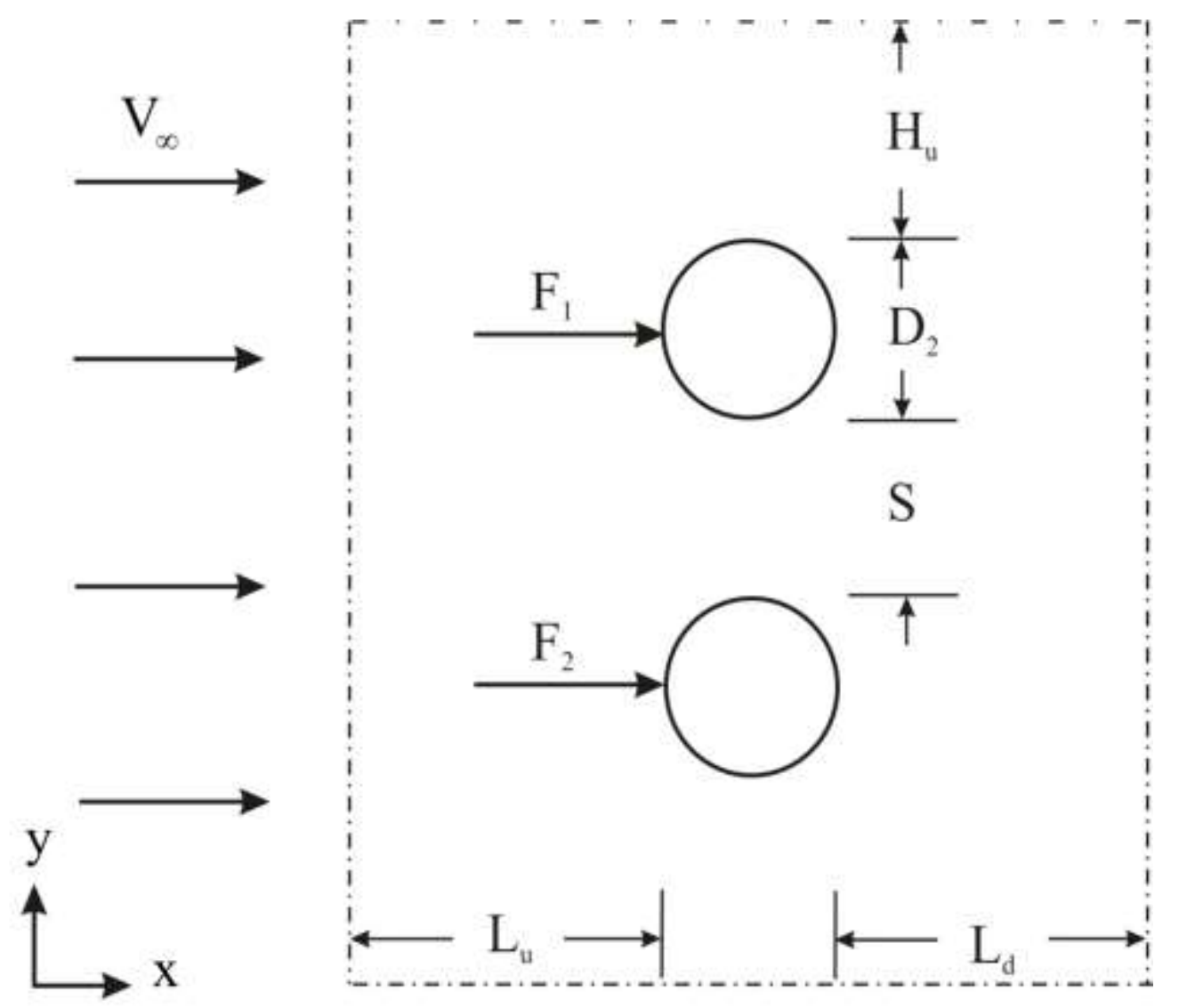

Fig. 4. Two parallel cylinders in cross flow.

The numerical model is built on the assumptions of unsteady incompressible flow in the laminar to transitional regime. The density and viscosity are assumed constant, which is a good model for the properties of water in the environmental range of temperature. The continuity and the time dependent conservation equations for momentum are

$$
\begin{aligned}
& \frac{\partial \mathrm{u}}{\partial \mathrm{x}}+\frac{\partial \mathrm{v}}{\partial \mathrm{y}}=0 \\
& \frac{\partial \mathrm{u}}{\partial \mathrm{t}}+\mathrm{u} \frac{\partial \mathrm{u}}{\partial \mathrm{x}}+\mathrm{v} \frac{\partial \mathrm{u}}{\partial \mathrm{y}}=-\frac{1}{\rho} \frac{\partial \mathrm{P}}{\partial \mathrm{x}}+v \nabla^{2} \mathrm{u} \\
& \frac{\partial \mathrm{v}}{\partial \mathrm{t}}+\mathrm{u} \frac{\partial \mathrm{v}}{\partial \mathrm{x}}+\mathrm{v} \frac{\partial \mathrm{v}}{\partial \mathrm{y}}=-\frac{1}{\rho} \frac{\partial \mathrm{P}}{\partial \mathrm{y}}+v \nabla^{2} \mathrm{v}
\end{aligned}
$$


where $\nabla^{2}=\partial^{2} / \partial \mathrm{x}^{2}+\partial^{2} / \partial \mathrm{y}^{2}$. The horizontal and vertical velocity components are $\mathrm{u}$ and $\mathrm{v}$, and the pressure is $\mathrm{P}$. The above equations were nondimensionalized based on the following dimensionless variables

$$
\begin{aligned}
& (\tilde{x}, \tilde{y}, \tilde{S})=\frac{(x, y, S)}{D}, \quad \tilde{t}=\frac{t V_{\infty}}{D} \\
& \tilde{P}=\frac{P}{\rho V_{\infty}^{2}}, \quad(\tilde{u}, \tilde{v})=\frac{(u, v)}{V_{\infty}},
\end{aligned}
$$

The resulting governing equations,

$$
\begin{aligned}
& \frac{\partial \tilde{\mathbf{u}}}{\partial \tilde{\mathrm{x}}}+\frac{\partial \tilde{\mathrm{v}}}{\partial \tilde{\mathrm{y}}}=0 \\
& \frac{\partial \tilde{\mathbf{u}}}{\partial \tilde{\mathrm{t}}}+\tilde{\mathrm{u}} \frac{\partial \tilde{\mathrm{u}}}{\partial \tilde{\mathrm{x}}}+\tilde{\mathrm{v}} \frac{\partial \tilde{\mathrm{u}}}{\partial \tilde{\mathrm{y}}}=-\frac{\partial \tilde{\mathrm{P}}}{\partial \tilde{\mathrm{x}}}+\frac{1}{\operatorname{Re}} \nabla^{2} \tilde{\mathrm{u}} \\
& \frac{\partial \tilde{\mathrm{v}}}{\partial \tilde{\mathrm{t}}}+\tilde{\mathrm{u}} \frac{\partial \tilde{\mathrm{v}}}{\partial \tilde{\mathrm{x}}}+\tilde{\mathrm{v}} \frac{\partial \tilde{\mathrm{v}}}{\partial \tilde{\mathrm{y}}}=-\frac{\partial \tilde{\mathrm{P}}}{\partial \tilde{\mathrm{y}}}+\frac{1}{\operatorname{Re}} \nabla^{2} \tilde{\mathrm{v}}
\end{aligned}
$$

show that the flow field depends on the Reynolds number

$$
\operatorname{Re}=\frac{\mathrm{V}_{\infty} \mathrm{D}}{v}
$$

The computational domain and the boundary conditions for fluid flow are shown in Fig. 4: $\tilde{\mathrm{u}}=1, \tilde{\mathrm{v}}=0$ at the inlet of the computational domain $(\tilde{\mathrm{x}}=0) ; \widetilde{\mathrm{P}}=0$ and $\partial(\tilde{\mathrm{u}}, \tilde{\mathrm{v}}) / \partial \tilde{\mathrm{x}}=0$ at the exit; no slip and no penetration on the cylinder surfaces; and $\tilde{\mathbf{v}}=0$ and $\partial \tilde{\mathbf{u}} / \partial \tilde{\mathbf{y}}=0$ on the top and bottom boundaries.

When $\operatorname{Re}$ is in the transitional range $(\operatorname{Re} \geq 30)$, the flow field undulates weakly and the two forces $\left(F_{1}, F_{2}\right)$ are not exactly the same. Therefore we calculated the sum of two forces, and formed the dimensionless total force exerted by the two cylinders: 


$$
\mathrm{f}=\frac{\mathrm{F}_{1}+\mathrm{F}_{2}}{\rho \mathrm{DV} \mathrm{V}_{\infty}^{2}}
$$

The dimensionless governing equations [Eqs. (16) - (18)] were solved by a commercial finite element code, Comsol. The domain was meshed with non-uniform mesh elements to grasp the effects of boundary layers, namely smaller mesh elements near the boundaries. In addition, adaptive mesh refinement was also used to decrease the effect of mesh element sizes throughout the solution time. Mesh tests were performed by increasing the number of the mesh elements in the steps of $50 \%$ until the effect on the results of $\mathrm{f}$ became less than $1 \%$. Because the spacing between the cylinders changed the size of the domain, the grid size varied for each case..

It was necessary to add virtual extensions to the computational domain, upstream, downstream and on the upper and lower boundaries, in order to account accurately for the pressure boundary conditions. These extensions $\left(\tilde{\mathrm{L}}_{\mathrm{u}}, \tilde{\mathrm{L}}_{\mathrm{d}}, \tilde{\mathrm{H}}_{\mathrm{u}}\right)$ are defined in Fig. 4. For example, the upper and lower extensions wereequal to $\tilde{\mathrm{H}}_{\mathrm{u}}$, which was selected to be large enough such that the results change less than 1 percent in $f$ when the $\tilde{H}_{u}$ extension was increased with a length equal to $\mathrm{D}_{2}$. We found that the extensions $\tilde{\mathrm{L}}_{\mathrm{u}}=4, \tilde{\mathrm{L}}_{\mathrm{d}}=5$ and $\tilde{\mathrm{H}}_{\mathrm{u}}=10$ were sufficiently large for the Re range covered by the numerical simulations when the domain consisted of two identical cylinders. Similarly, the time step of $\Delta \tilde{\mathrm{t}}=10^{3}$ was found to be short enough so that the effect of $\Delta \tilde{\mathrm{t}}$ on $\mathrm{f}$ is negligible.

\section{Numerical Results}

Figure 5 shows the effect of the spacing $\widetilde{\mathrm{S}}=\mathrm{S} / \mathrm{D}$ on the total force on the two cylinders.

The value of f at $\tilde{\mathrm{S}}=0$, is approximately 2.7 (check value). This seems to be correct as from Eq. 3, this value is determined from $C_{A} n$ where $C_{A}$ is in the order of 1.35 and $n=2$. An optimal 
spacing emerges close to $\widetilde{S}=0.6$ (note: to me - it looks closer to 0.5 or 0.5 to 0.6 This value depends on the Reynolds number, as shown in Fig. 6 . When $\operatorname{Re} \geq 50$, the $f$ values oscillate weakly (within 3 percent) as Re increases. These numerical simulations allow us to estimate not only the maximum force but also the force in the limit of cylinders stuck together, $f_{0}=f(\widetilde{S}=0)$, and the force in the limit of large spacing, $f_{\infty}=f(\tilde{S}>>1)$. All three forces depend on Re.

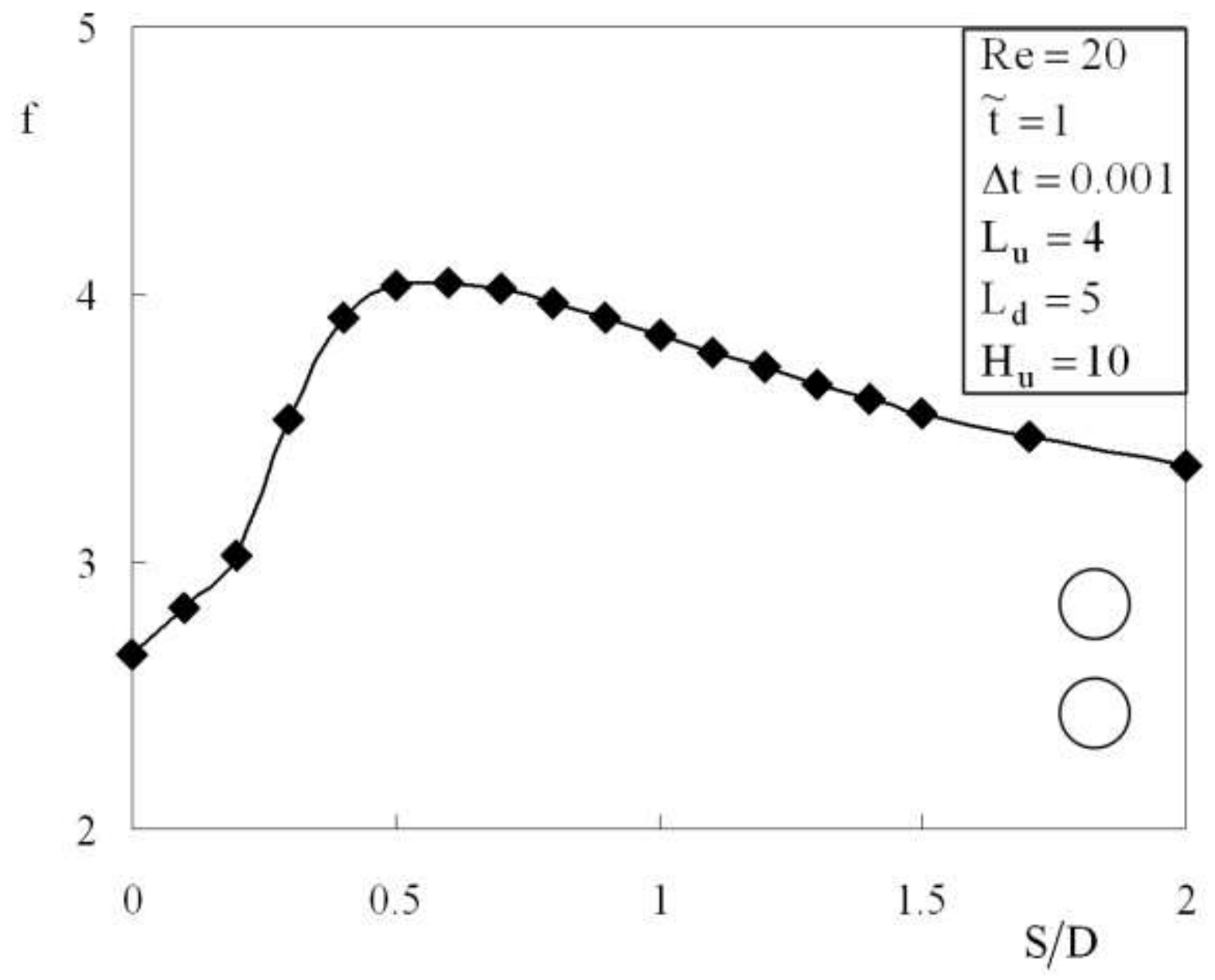

Fig. 5. The effect of the spacing on the total force exerted by the two cylinders. 


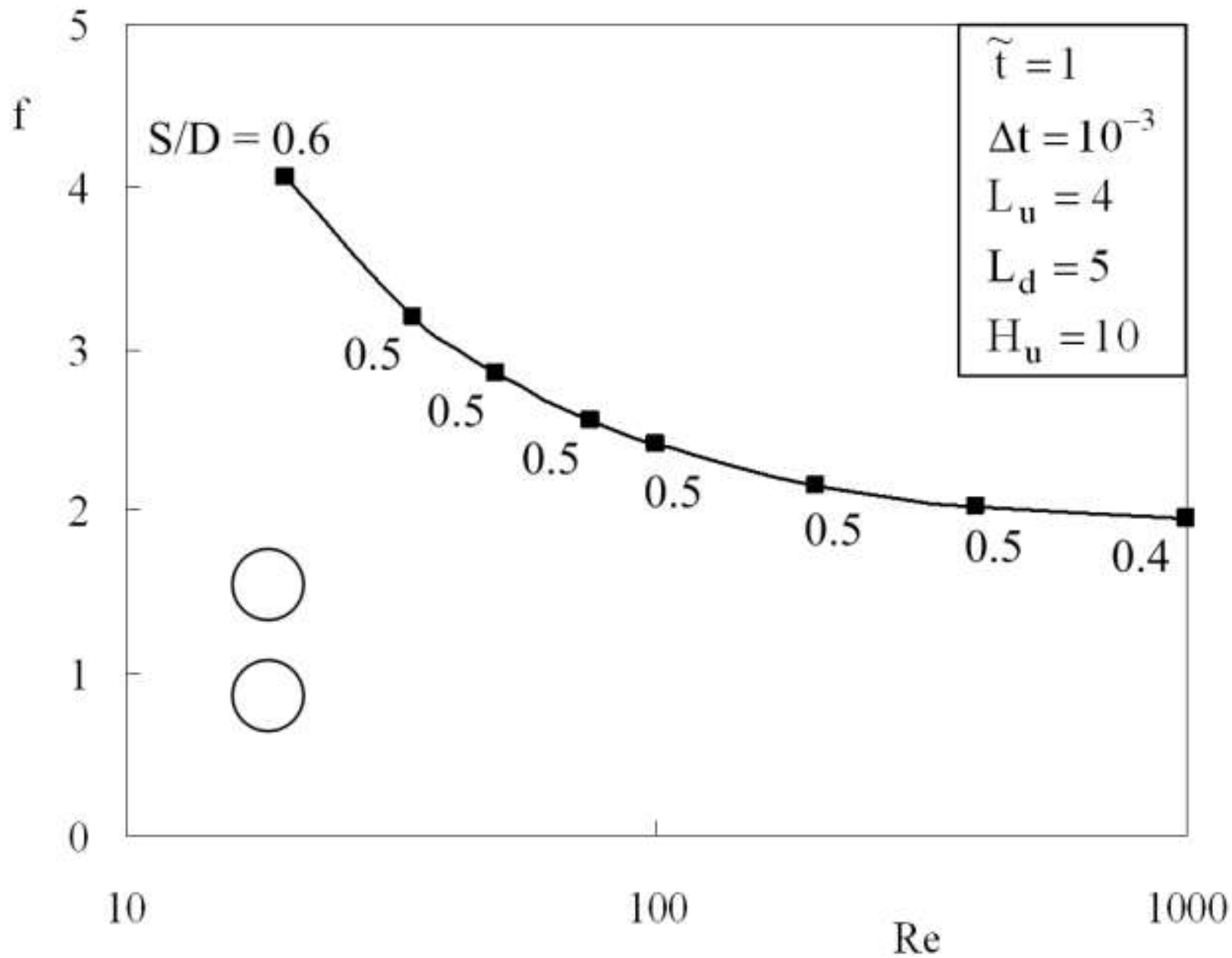

Fig. 6. The Re effect on the optimal spacing and the maximum total force, two cylinders.

Important is also the gain $(\mathrm{g})$ in force associated with using the optimal spacing relative to zero spacing,

$$
\mathrm{g}_{0}=\frac{\mathrm{f}_{\max }-\mathrm{f}_{0}}{\mathrm{f}_{0}}
$$

which for $\operatorname{Re}=20$ has the value $\mathrm{g}_{0}=0.53$. The gain in force relative to sufficiently wide spacings,

$$
g_{\infty}=\frac{f_{\max }-f_{\infty}}{f_{\infty}}
$$

is $\mathrm{g}_{\infty}=0.38$ for $\mathrm{Re}=20$. When Re number increases, $\mathrm{g}_{0}$ decreases and $\mathrm{g}_{\infty}$ increases such that $\mathrm{g}_{0}$ 
$=0.44$ and $\mathrm{g}_{\infty}=0.52$ when $\operatorname{Re}=100$. These gains $\left(\mathrm{g}_{0}, \mathrm{~g}_{\infty}\right)$ are significant and only weakly dependent on Re.
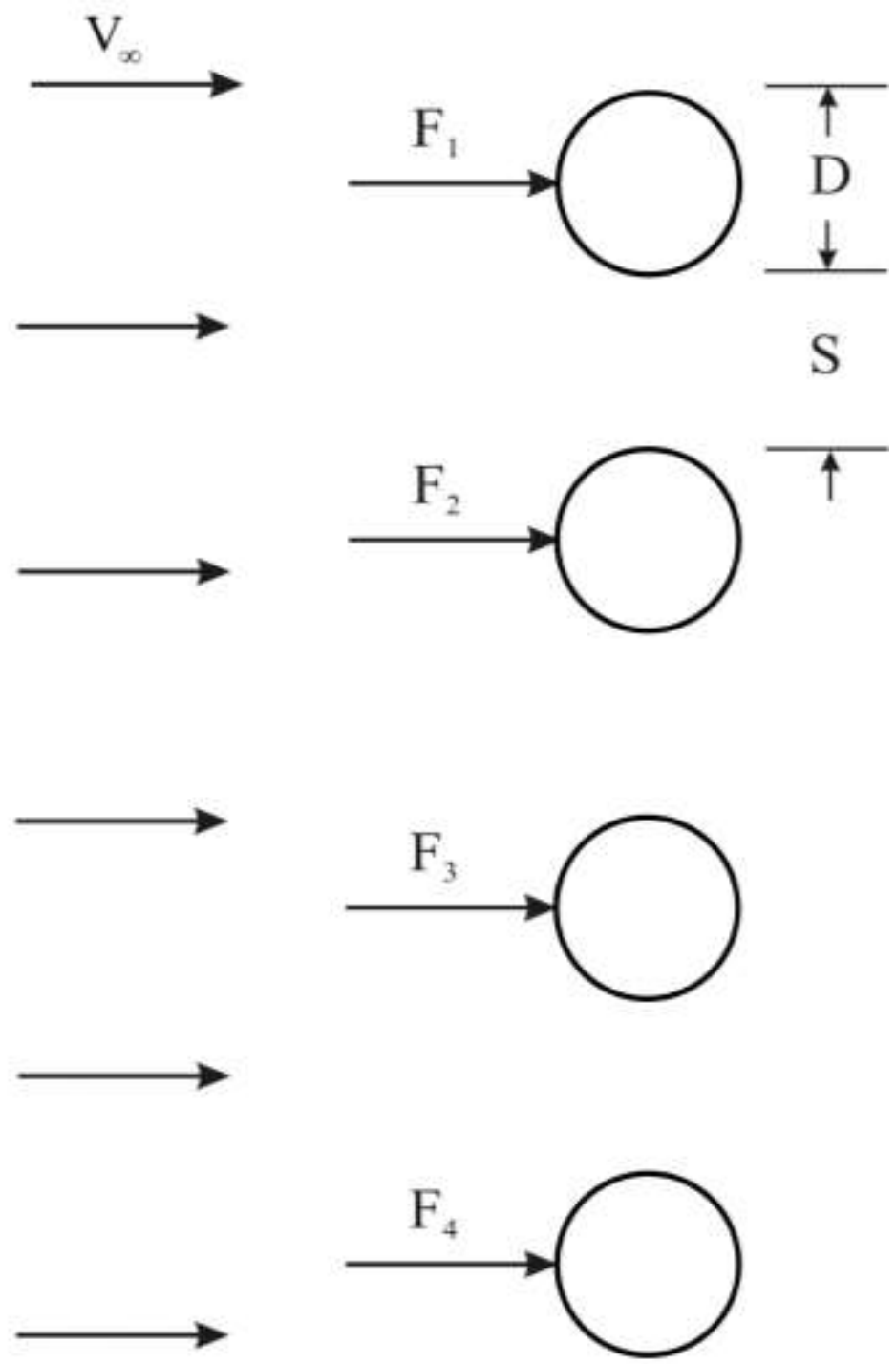

Fig. 7. Four parallel cylinders in cross flow.

A more realistic model of paddling foot or palm is the four parallel cylinders shown in Fig. 7. We used the same method as for two cylinders, and produced results that correspond to Fig. 5, to document the effect of $\mathrm{S}$ on $\mathrm{f}$ at fixed Re. The total force is defined as 


$$
\mathrm{f}=\frac{\mathrm{F}_{1}+\mathrm{F}_{2}+\mathrm{F}_{3}+\mathrm{F}_{4}}{\rho \mathrm{DV} \mathrm{V}_{\infty}^{2}}
$$

Figure 8 shows the effect of Re number on the maximum total force and the optimal spacing that corresponds to the maximum total force on four cylinders. These results reinforce the conclusion reached earlier by simulating the force on only two cylinders (Fig. 6). The total force felt by four cylinders is roughly three times the force felt by two cylinders. The optimal spacing (S/D approx. equal 0.4) for four cylinders is almost the same as the optimal spacing (S/D approx. equal to 0.5) for two cylinders. This validates the prediction offered in Eq. (9), where the optimal spacing depends solely on the diameter (D) of two adjacent cylinders. Furthermore, Eq. (9) anticipates that S/D should decrease as Re increases, and this trend is also validated by the numerical results.

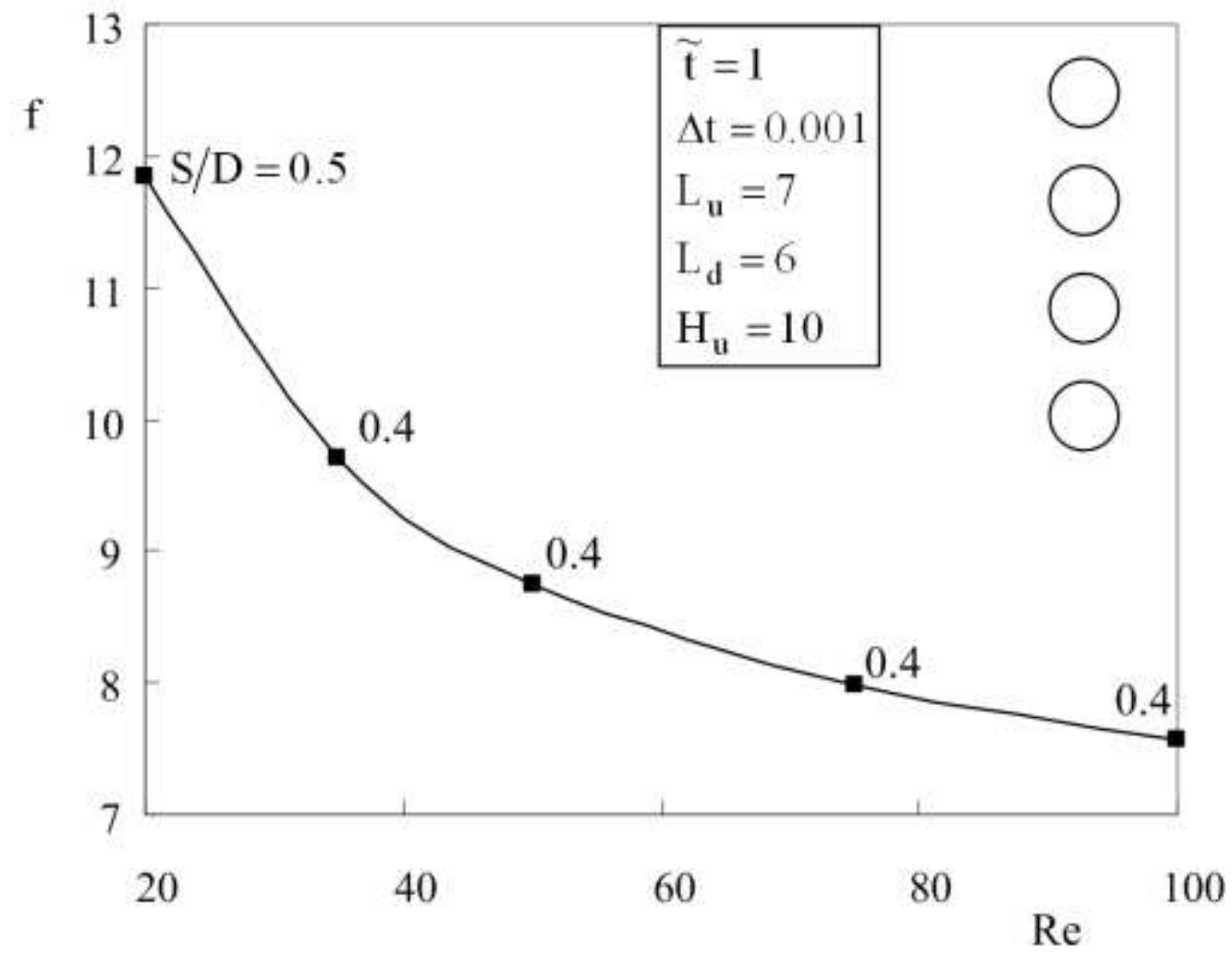

Fig. 8. The Re effect on the optimal spacing and the maximum total force, four cylinders. 
Figure 9 shows the total force exerted by four cylinders relative to S/D when the flow is steady. As in transient flow regime (Fig. 8), an optimal spacing emerges in the steady flow regime, which corresponds to the $\mathrm{t} \rightarrow \infty$ limit of the unsteady flows of the preceding examples. Fig. 10 is a summary of the maximum forces and optimal spacings in the steady flow regime. Together, Fig. 8, Fig. 9 and Fig. 10 show that the optimal spacing phenomenon emerges in both regimes, transient or steady. Fig. 11 shows the time effect on the maximum total force and the spacing that corresponds to the maximum total force. The maximum total force and the optimal spacing oscillate weakly, and the oscillation becomes weaker as the time increases.

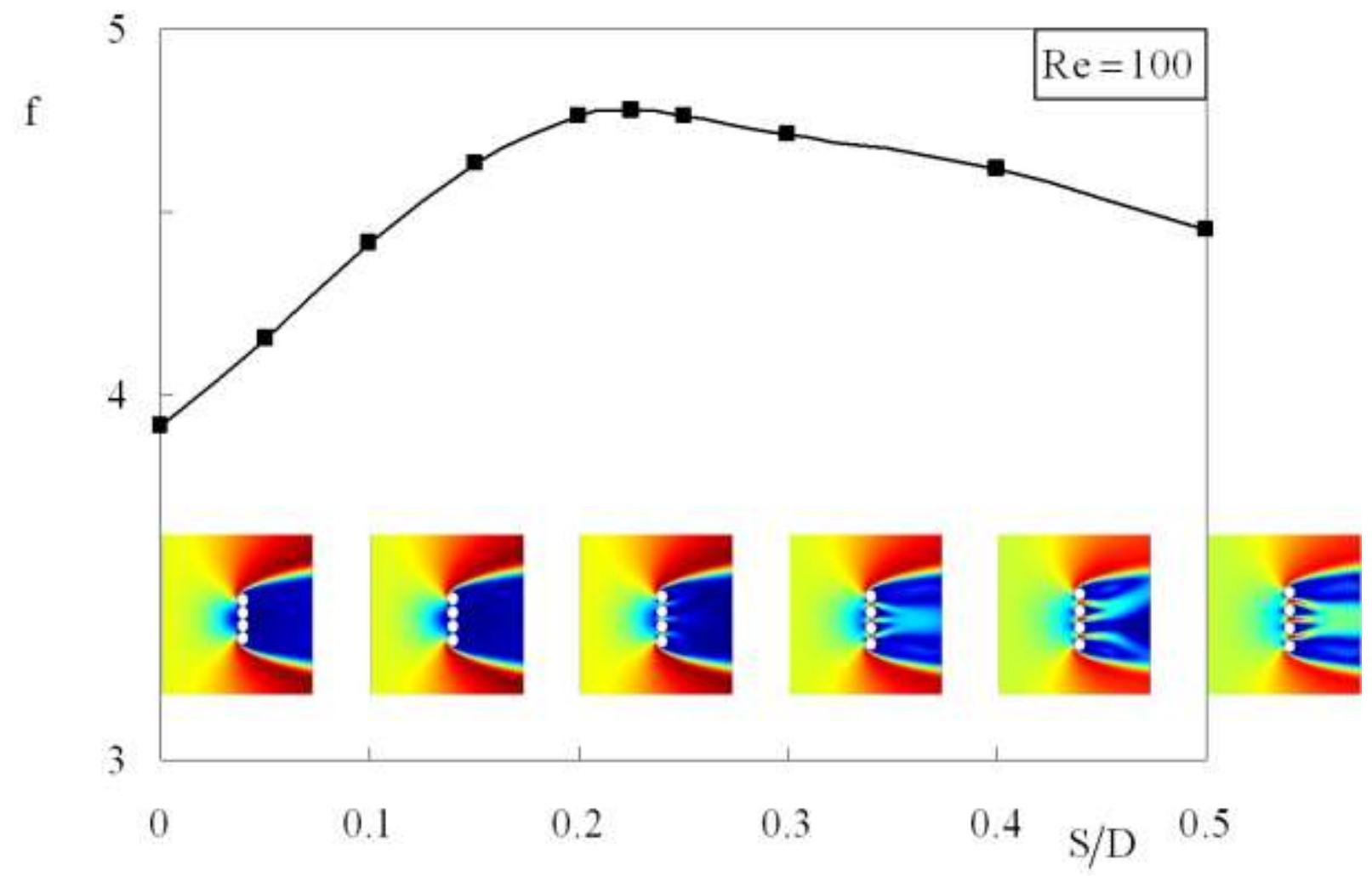

Fig. 9. The effect of the spacing on the total force when the flow regime is steady. 


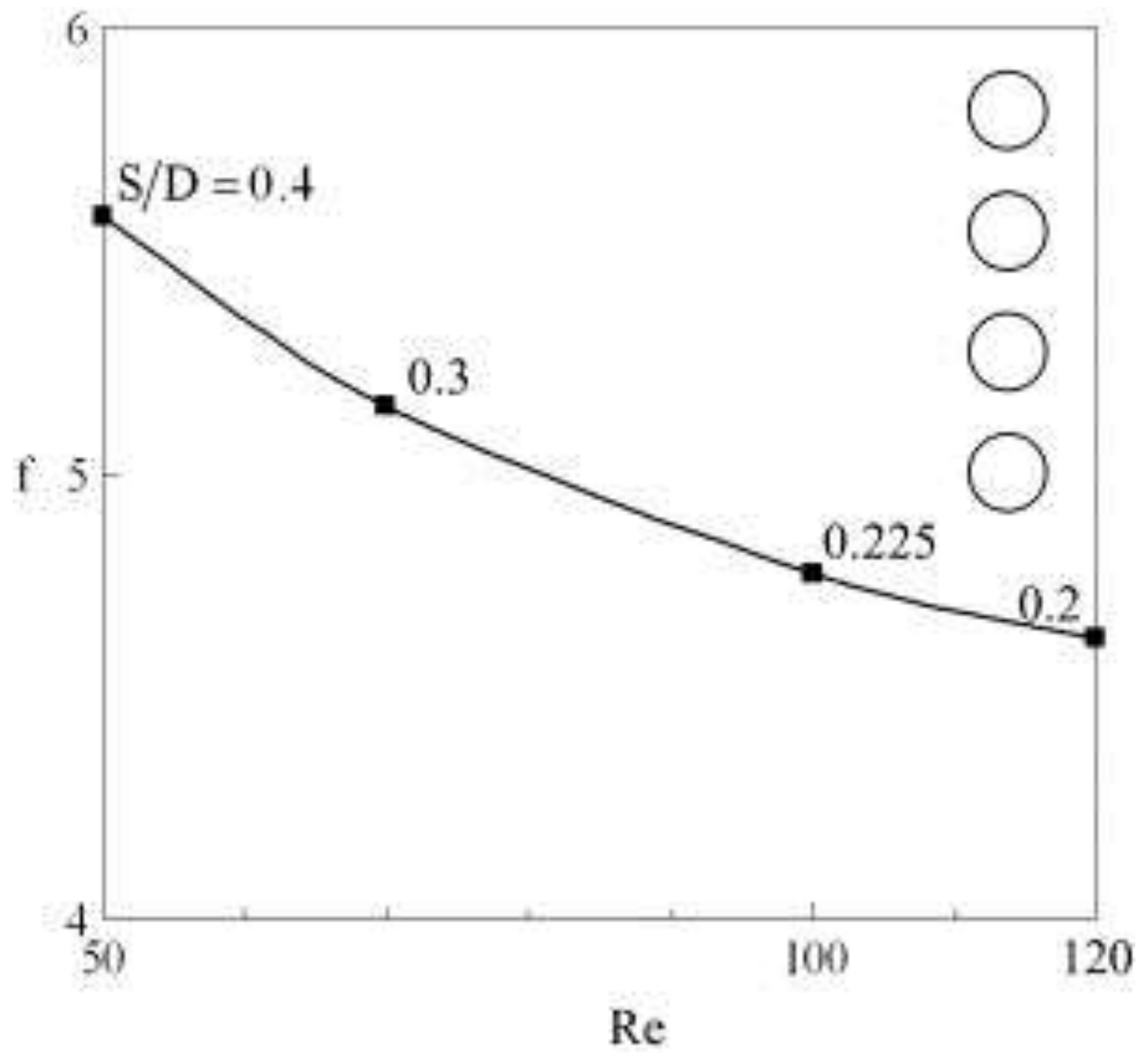

Fig. 10. The optimal spacing and total force when the flow regime is steady. 


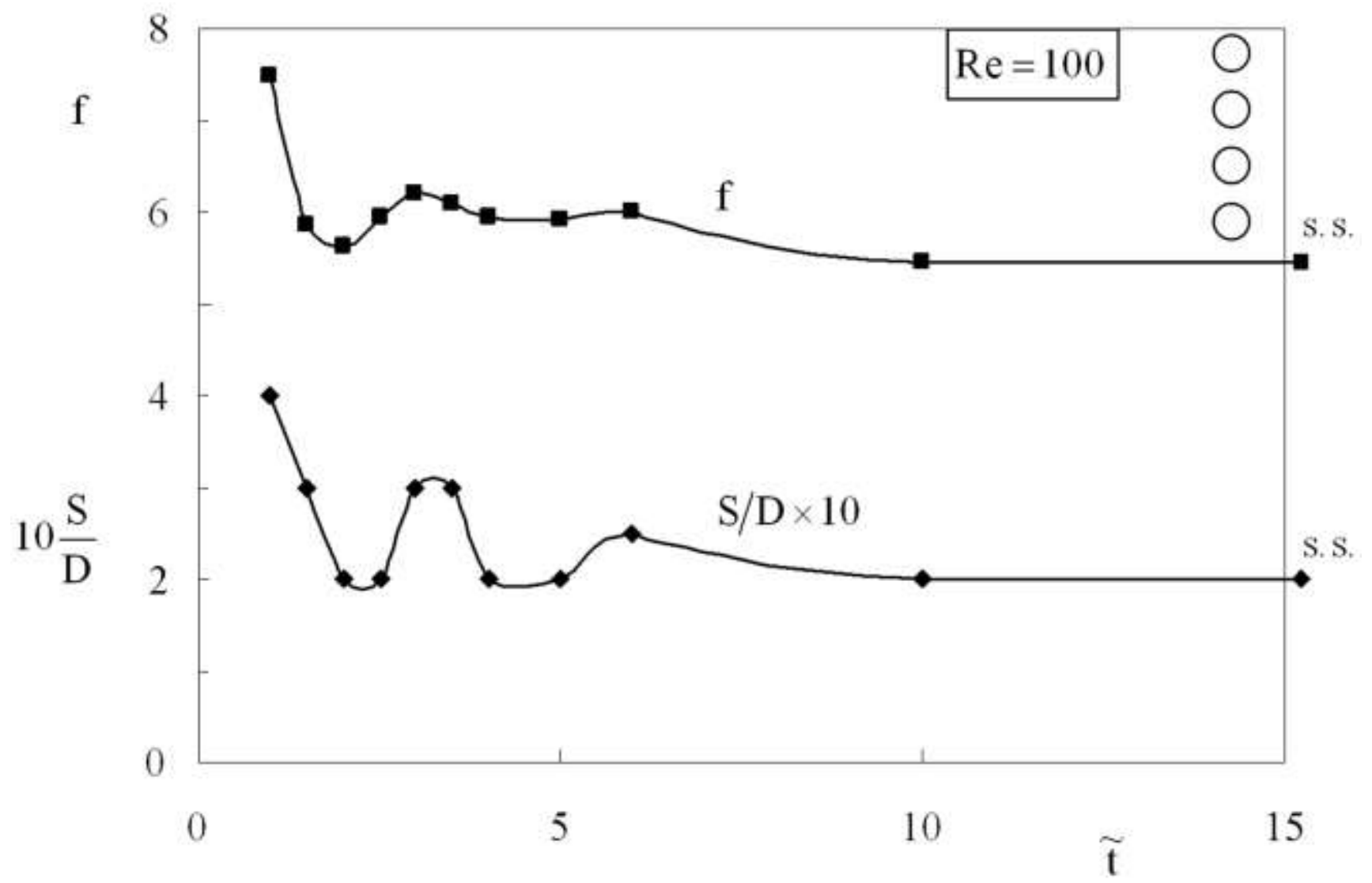

Fig. 11. The effect of time on the total force and the optimal spacing, $R e=100$.

\section{Conclusions}

In this paper we showed theoretically that there exists an optimal spacing between fingers such that the force exerted by the hand on water is maximal during swimming (section 2). The maximization of force is an integral feature of the evolutionary design of swimming animals and athletes for survival and speed (section 1).

The existence of the optimal spacing was confirmed by computational simulations of water flowing frontally on two and four cylinders in cross flow. In the Re range $20-100$, we found that the optimal spacing is in the range $0.2 \mathrm{D}-0.4 \mathrm{D}$, and decreases slowly as Re increases. This spacing is of the same order as the finger-to-finger spacing practiced by competitive swimmers. 
Even though the flow across cylinders is time dependent, the optimal spacing persists in the limit $\tilde{\mathrm{t}}>>1$ and in the steady state (s.s., Fig. 10). Optimally spaced fingers and toes are the natural design for efficient swimming paddles without web. The webbed foot (Fig. 1) is a subsequent design feature that enhances the force-augmentation effect, in the way that the drag coefficient jumps from $\mathrm{C}_{2}$ to $\mathrm{C}_{3}$ in Fig. 2 .

\section{Acknowledgement}

This research was supported by the National Science Foundation, the Air Force Office of Scientific Research, and the Republic of Turkey.

\section{References}

Bejan, A., Lorente, S., 2008. Design with Constructal Theory. Wiley, Hoboken (Chapter 3)

Bejan, A., Lorente, S., 2010. The constructal law of design and evolution in nature. Phil. Trans. Royal Soc. B 365, 1335 - 1347.

Bejan, A., Marden, J. H., 2006. Unifying Constructal theory for scale effects in running, swimming and flying. J. Exp. Biol. 209, 238-248.

Bejan, A., Jones, E. C., Charles, J. D., 2010. The evolution of speed in athletics: why the fastest runners are black and swimmers white. Int. J. Design and Nature Ecodyn. 5(3), 199 - 211.

Bixler, B. Riewald, S., 2002. Analysis of a swimmer's hand and arm in steady flow conditions using computational dynamics. J. Biomech. 35, 713-717.

Charles, J. D., Bejan, A., 2009. The evolution of speed, size and shape in modern athletics. J. Exp. Biol. 212, 2419-2425.

Hoppeler, H., Weibel, E. R., 2005. Scaling functions to body size: theories and facts, special issue. J. Exp. Biol. 208, 1573 - 1769. 
Kudo, S., Vennell, R., Wilson, B., Waddell, N., Sato, Y., 2008. Influence of surface penetration on measured fluid force on a hand model. J. Biomech. 41

Marinho, D. A., Barbosa, T. M. Reis, V. M., Kjendlie, P. L., Alves, F. B., Vilas - Boas, J. P., Machado, L., Silva, A. J., Rouboa, A. I., 2010. Swimming propulsion forces are enhanced by a small finger spread. J. Appl. Biomech. 26, 87 - 92 .

Miguel, A. F., 2006. Constructal pattern formation in strong corals, bacterial colonies and plant roots under different hydrodynamics conditions. J. Theor. Biol. 242, 954 -961.

Minetti, A. E., Machtsiras, G., Masters, J. C., 2009. The optimum finger spacing in human swimming. J. Biomech. 42, 2188-2190.

Reis, A. H., 2006a. Constructal view of scaling laws of river basins. Geomorphology 78, $201-$ 206.

Reis, A. H., 2006b. Constructal theory: from engineering to physics, and how flow systems develop shape and structure. Appl. Mech. Rev. 59, 269 - 282.

Reis, A. H., Miguel, A. F., Aydin, M., 2004. Constructal theory of flow architecture of the lungs. Med. Phys. 31, $1135-1140$.

Rouboa, A., Silva, A., Leal, L., Rocha J., Alves, F., 2006. The effect of swimmer's hand/forearm acceleration on propulsive forces generation using computational fluid dynamics. J. Biomech. 39, 1239 - 1248.

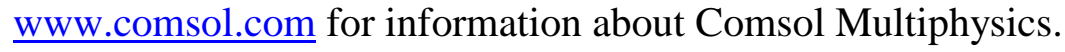

